\title{
Minimizing Indices of Conditional Expectations onto a Subfactor
}

By

\author{
Fumio HIAI*
}

\begin{abstract}
For a pair of factors $M \supseteq N$, let $\mathscr{E}(M, N)$ be the set of all conditional expectations from $M$ onto $N$. We characterize $E_{0} \in \mathscr{E}(M, N)$ whose index is the minimum of \{Index $E: E \in$ $\mathscr{E}(M, N)\}$. When $M \supseteq N$ are $\mathrm{II}_{1}$ factors, we establish the relation between Index $E_{0}$ and $[M: N]$.
\end{abstract}

\section{Introduction}

Jones [5] developed the index theory for type $\mathrm{II}_{1}$ factors using the coupling constant and Umegaki's conditional expectation [10]. Kosaki [6] extended it to arbitrary factors. Let $M$ be a factor and $N$ a subfactor of $M$. We denote by $\mathscr{E}(M, N)$ the set of all faithful normal conditional expectations from $M$ onto $N$. The index Index $E$ of $E \in \mathscr{E}(M, N)$ was introduced in [6] based on Connes' spatial theory [3] and Haagerup's theory on operator valued weights [4] as follows: Index $E=E^{-1}(1)$ where $E^{-1}$ is the operator valued weight from $N^{\prime}$ to $M^{\prime}$ characterized by the equation $d(\varphi \circ E) / d \psi=d \varphi / d\left(\psi \circ E^{-1}\right)$ of spatial derivatives. Here $\varphi$ and $\psi$ are faithful normal semifinite weights on $N$ and $M^{\prime}$, respectively. See also $[9,12.11]$.

As shown in [2, Théorème 1.5.5], $\mathscr{E}(M, N)$ contains at most one element if the relative commutant $N^{\prime} \cap M$ is $C 1$. But $\mathscr{E}(M, N)$ has many elements in general. Indeed, when $\mathscr{E}(M, N) \neq \emptyset$, the map $E \mapsto E \mid N^{\prime} \cap M$ is a bijection from $\mathscr{E}(M, N)$ onto the set of all faithful normal states on $N^{\prime} \cap M$ (see [1, Théorème 5.3]). The aim of this paper is to discuss the problem when Index $E$ takes

Communicated by H. Araki, February 24, 1988.

* Division of Applied Mathematics, Research Institute of Applied Electricity, Hokkaido University, Sapporo 060, Japan. 
the minimum value for a fixed pair $M \supseteq N$.

\section{\& 1. Main Result}

From now on, let $M$ be a $\sigma$-finite factor and $N$ a subfactor of $M$ with $\mathscr{E}(M, N) \neq \emptyset$. We first note the following two facts:

$1^{\circ}$ If Index $E<4$ for some $E \in \mathscr{E}(M, N)$, then $N^{\prime} \cap M=C$ 1 (see [6, Theorem 4.4]). In this case, $\mathscr{E}(M, N)$ consists of one element. $2^{\circ}$ If Index $E<\infty$ for some $E \in \mathscr{E}(M, N)$, then $N^{\prime} \cap M$ is finite dimensional (see [6, Proposition 4.3]). In this case, since (Index $E)^{-1} E^{-1} \in \mathscr{E}\left(N^{\prime}, M^{\prime}\right)$, it follows from [4, Theorem 6.6] that each operator valued weight from $N^{\prime}$ to $M^{\prime}$ is bounded. Hence Index $E^{\prime}<\infty$ for every $E^{\prime} \in \mathscr{E}(M, N)$.

The fact $2^{\circ}$ shows that either Index $E<\infty$ for all $E \in \mathscr{E}(M, N)$ or Index $E=\infty$ for all $E \in \mathscr{E}(M, N)$.

Theorem 1. Assume that Index $E<\infty$ for some (hence all) $E \in$ $\mathscr{E}(M, N)$.

(1) There exists a unique $E_{0} \in \mathscr{E}(M, N)$ such that

Index $E_{0}=\min \{$ Index $E: E \in \mathscr{E}(M, N)\}$.

(2) If $E \in \mathscr{E}(M, N)$, then the following conditions are equivalent:

(i) $E=E_{0}$;

(ii) $E \mid N^{\prime} \cap M$ and $E^{-1} \mid N^{\prime} \cap M$ are traces and

$$
E^{-1} \mid N^{\prime} \cap M=(\text { Index } E) E \mid N^{\prime} \cap M ;
$$

(iii) $E^{-1}\left|N^{\prime} \cap M=c E\right| N^{\prime} \cap M$ for some constant $c$.

(3) If $N^{\prime} \cap M \neq C l$, then

$\{$ Index $E: E \in \mathscr{E}(M, N)\}=\left[\right.$ Index $\left.E_{0}, \infty\right)$.

Proof. We first show that there exists an $E_{0} \in \mathscr{E}(M, N)$ satisfying condition (ii) of (2). Let $\varphi$ and $\psi$ be faithful normal semifinite weights on $N$ and $M^{\prime}$, respectively. By [1, Théorème 5.3], we can choose an $E \in \mathscr{E}(M, N)$ such that $E \mid N^{\prime} \cap M$ is a trace. For every unitary $u$ in $N^{\prime} \cap M$, we have by [3, Proposition 8 and Theorem 9]

$$
\begin{aligned}
\frac{d\left(\varphi \circ u E u^{*}\right)}{d \psi} & =u \frac{d(\varphi \circ E)}{d \psi} u^{*} \\
& =\left(u \frac{d\left(\varphi \circ E^{-1}\right)}{d \varphi} u^{*}\right)^{-1}
\end{aligned}
$$




$$
=\frac{d \varphi}{d\left(\psi \circ u E^{-1} u^{*}\right)},
$$

where $u E u^{*}=E\left(u^{*} \cdot u\right)$. Hence $\left(u E u^{*}\right)^{-1}=u E^{-1} u^{*}$. Since $u E u^{*} \mid N^{\prime} \cap M$ $=E \mid N^{\prime} \cap M$, we get $u E u^{*}=E$ by $[1$, Théorème 5.3] again, so that $u E^{-1} u^{*}=E^{-1}$. This shows that $E^{-1} \mid N^{\prime} \cap M$ is a trace. Choosing minimal projections $f_{1}, \cdots, f_{n}$ in $N^{\prime} \cap M$ with $\sum_{i} f_{i}=1$, we define a positive invertible element $h$ in the center of $N^{\prime} \cap M$ by $h=\sum_{i=1}^{n} \alpha_{i} f_{i}$ where

$$
\alpha_{i}=\left(\sum_{i=1}^{n} E\left(f_{i}\right)^{1 / 2} E^{-1}\left(f_{i}\right)^{1 / 2}\right)^{-1} \frac{E^{-1}\left(f_{i}\right)^{1 / 2}}{E\left(f_{i}\right)^{1 / 2}}, \quad 1 \leqq i \leqq n .
$$

Now let $E_{0}=h^{1 / 2} E h^{1 / 2}$. Then $E_{0} \in \mathscr{E}(M, N)$ follows from

$$
E_{0}(y)=E(h y)=E(h) y=y, \quad y \in N .
$$

Since

$$
\begin{aligned}
\frac{d\left(\varphi \circ E_{0}\right)}{d \psi} & =h^{1 / 2} \frac{d(\varphi \circ E)}{d \psi} h^{1 / 2} \\
& =\left(h^{-1 / 2} \frac{d\left(\psi \circ E^{-1}\right)}{d \varphi} h^{-1 / 2}\right)^{-1} \\
& =\frac{d \varphi}{d\left(\psi \circ h^{-1 / 2} E^{-1} h^{-1 / 2}\right)},
\end{aligned}
$$

we get $E_{0}^{-1}=h^{-1 / 2} E^{-1} h^{-1 / 2}$ and hence

$$
\frac{E_{0}^{-1}\left(f_{i}\right)}{E_{0}\left(f_{i}\right)}=\frac{E^{-1}\left(h^{-1} f_{i}\right)}{E\left(h f_{i}\right)}=\alpha_{i}^{-2} \frac{E^{-1}\left(f_{i}\right)}{E\left(f_{i}\right)}=c, \quad 1 \leqq i \leqq n,
$$

where $c=\left(\sum_{i=1}^{n} E\left(f_{i}\right)^{1 / 2} E^{-1}\left(f_{i}\right)^{1 / 2}\right)^{2}$. Therefore $E_{0}^{-1}\left|N^{\prime} \cap M=c E_{0}\right| N^{\prime} \cap M$, so that $c=\operatorname{Index} E_{0}$.

(1) For each $E \in \mathscr{E}(M, N)$, let $h$ be the Radon-Nikodym derivative of $E \mid N^{\prime} \cap M$ with respect to the trace $E_{0} \mid N^{\prime} \cap M$. Since $E=$ $h^{1 / 2} E_{0} h^{1 / 2}$ follows from $E\left|N^{\prime} \cap M=h^{1 / 2} E_{0} h^{1 / 2}\right| N^{\prime} \cap M$, we obtain $E^{-1}=$ $h^{-1 / 2} E_{0}^{-1} h^{-1 / 2}$ as above. Hence

$$
\text { Index } \begin{aligned}
E & =E_{0}^{-1}\left(h^{-1}\right) \\
& =\left(\operatorname{Index} E_{0}\right) E_{0}\left(h^{-1}\right) \\
& \geqq \operatorname{Index} E_{0},
\end{aligned}
$$

because

$$
\mathrm{l}=E_{0}(1) \leqq E_{0}(h)^{1 / 2} E_{0}\left(h^{-1}\right)^{1 / 2}=E_{0}\left(h^{-1}\right)^{1 / 2} .
$$

Moreover it is readily checked that $E_{0}\left(h^{-1}\right)=1$ holds if and only if $h=1$, i. e. $E=E_{0}$. Therefore (1) is proved. 
(2) (i) $\Rightarrow$ (ii) is seen from the construction of $E_{0}$, and (ii) $\Rightarrow$ (iii) is trivial. To show (iii) $\Rightarrow$ (i), assume that $E \in \mathscr{E}(M, N)$ satisfies (iii). Then $c=\operatorname{Index} E$. Let $h$ be as in the proof of (1). Because $E_{0}^{-1}=$ $h^{1 / 2} E^{-1} h^{1 / 2}$ and

$$
\text { Index } \begin{aligned}
E_{0} & =E^{-1}(h) \\
& =(\operatorname{Index} E) E(h) \\
& =(\operatorname{Index} E) E_{0}\left(h^{2}\right) \\
& \geqq\left(\operatorname{Index} E_{0}\right) E_{0}\left(h^{2}\right),
\end{aligned}
$$

we get

$$
E_{0}\left((h-1)^{2}\right)=E_{0}\left(h^{2}\right)-1 \leqq 0,
$$

implying $h=1$ and thus $E=E_{0}$.

(3) Assuming $N^{\prime} \cap M \neq C$ l, we choose nonzero projections $p_{1}$ and $p_{2}$ in $N^{\prime} \cap M$ with $p_{1}+p_{2}=1$. For each $h=\alpha_{1} p_{1}+\alpha_{2} p_{2}$ with $\alpha_{1}, \alpha_{2}>0$ and $\alpha_{1} E_{0}\left(p_{1}\right)+\alpha_{2} E_{0}\left(p_{2}\right)=1$, letting $E=h^{1 / 2} E_{0} h^{1 / 2}$ we obtain $E \in$ $\mathscr{E}(M, N)$ and

$$
\text { Index } \begin{aligned}
E & =E_{0}^{-1}\left(h^{-1}\right) \\
& =\left(\operatorname{Index} E_{0}\right)\left(\alpha_{1}^{-1} E_{0}\left(p_{1}\right)+\alpha_{2}^{-1} E_{0}\left(p_{2}\right)\right) .
\end{aligned}
$$

Therefore Index $E$ can take any real numbers in [Index $\left.E_{0}, \infty\right)$.

\section{§2. Case of $\mathrm{II}_{1}$ Factors}

Now let $M$ be a type $\mathrm{II}_{1}$ factor with the normalized trace $\tau$. For a subfactor $N$ of $M$, let $E_{N} \in \mathscr{E}(M, N)$ be Umegaki's conditional expectation [10] with respect to $\tau$. Then Index $E_{N}$ coincides with Jones' index $[M: N]$ (see [6]). By definition of Jones' index [5], $[M: N]<\infty$ if and only if $N^{\prime}$ on $L^{2}(M, \tau)$ is finite. In this case, let $\tau^{\prime}$ be the normalized trace on $N^{\prime}$.

Theorem 2. Let $M \supseteq N$ be factors of type $\mathrm{II}_{1}$ with $[M: N]<\infty$, and $f_{1}, \cdots, f_{n}$ be minimal projections in $N^{\prime} \cap M$ with $\sum_{i} f_{i}=1$.

(1) If $E_{0} \in \mathscr{E}(M, N)$ is as in Theorem 1, then

$$
\text { Index } E_{0}=[M: N]\left(\sum_{i=1}^{n} \tau\left(f_{i}\right)^{1 / 2} \tau^{\prime}\left(f_{i}\right)^{1 / 2}\right)^{2} .
$$

(2) The following conditions are equivalent:

(i) $[M: N]=\min \{$ Index $E: E \in \mathscr{E}(M, N)\}$; 
(ii) $\tau^{\prime}\left|N^{\prime} \cap M=\tau\right| N^{\prime} \cap M$.

(3) If $[M: N] \geqq 4$, then

$$
[M: N] \geqq 4\left(\sum_{i=1}^{n} \tau\left(f_{i}\right)^{1 / 2} \tau^{\prime}\left(f_{i}\right)^{1 / 2}\right)^{-2} .
$$

Proof. (1) We can take $E=E_{N}$ in the first part of the proof of Theorem 1. Since $E_{N}\left|N^{\prime} \cap M=\tau\right| N^{\prime} \cap M$ and $E_{N}^{-1} \mid N^{\prime} \cap M=[M: N]$ $\tau^{\prime} \mid N^{\prime} \cap M$ (see [6]), we have $E_{0}=h^{1 / 2} E_{N} h^{1 / 2}$ where $h=\sum_{i=1}^{n} \alpha_{i} f_{i}$ and

$$
\alpha_{i}=\left(\sum_{i=1}^{n} \tau\left(f_{i}\right)^{1 / 2} \tau^{\prime}\left(f_{i}\right)^{1 / 2}\right)^{-1} \frac{\tau^{\prime}\left(f_{i}\right)^{1 / 2}}{\tau\left(f_{i}\right)^{1 / 2}}, \quad 1 \leqq i \leqq n .
$$

Therefore

$$
\begin{aligned}
\text { Index } E_{0} & =E_{N}^{-1}\left(h^{-1}\right) \\
& =[M: N] \sum_{i=1}^{n} \alpha_{i}^{-1} \tau^{\prime}\left(f_{i}\right) \\
& =[M: N]\left(\sum_{i=1}^{n} \tau\left(f_{i}\right)^{1 / 2} \tau^{\prime}\left(f_{i}\right)^{1 / 2}\right)^{2} .
\end{aligned}
$$

(2) Because condition (i) means $E_{N}=E_{0}$, it follows from Theorem 1 (2) that (i) is equivalent to $E_{N}^{-1}\left|N^{\prime} \cap M=[M: N] E_{N}\right| N^{\prime} \cap M$, that is, $\tau^{\prime}\left|N^{\prime} \cap M=\tau\right| N^{\prime} \cap M$.

(3) Since $[M: N] \geqq 4$, we get Index $E_{0} \geqq 4$ (see $1^{\circ}$ before Theorem 1). Then the desired inequality follows from (1).

Remarks. Let $M \supseteq N$ be type $\mathrm{II}_{1}$ factors with $[M, N]<\infty$.

(1) Let $\mathrm{H}(M \mid N)$ be the entropy considered in [7]. It was shown in [7, Corollary 4.5] that condition (ii) of Theorem 2 is equivalent to the equality $\mathrm{H}(M \mid N)=\log [M: N]$. In particular if $[M: N]=4$, then $\sum_{i} \tau\left(f_{i}\right)^{1 / 2} \tau^{\prime}\left(f_{i}\right)^{1 / 2}=1$ by Theorem $2(3)$, so that (ii) holds. In this connection, see [7, Corollary 4.8].

(2) Let $N \subseteq M \subseteq M_{1} \subseteq M_{2} \subseteq \cdots$ be the sequence of type $\mathrm{II}_{1}$ factors obtained by iterating the basic construction [5]. The following result is in [8]: If $\mathrm{H}(M \mid N)=\log [M: N]$ (equivalently $[M: N]=\min$ $\{$ Index $E: E \in \mathscr{E}(M, N)\}$ ), then $\mathrm{H}\left(M_{n} \mid N\right)=\log \left[M_{n}: N\right]$ (equivalently $\left.\left[M_{n}: N\right]=\min \left\{\operatorname{Index} E: E \in \mathscr{E}\left(M_{n}, N\right)\right\}\right)$ for every $n \geqq 1$.

\section{References}

[1] Combes, F. and Delaroche, C., Groupe modulaire d'une espérance conditionnelle dans 
une algèbre de von Neumann, Bull. Soc. Math. France, 103 (1975), 385-426.

[2] Connes, A., Une classification des facteurs de type III, Ann. Sci. École Norm. Sup. Sér. 4, 6 (1973), 133-252.

[3] , On the spatial theory of von Neumann algebras, J. Funct. Anal., 35 (1980), 153-164.

[4] Haagerup, U., Operator valued weights in von Neumann algebras, I, II, J.Funct. Anal., 32 (1979), 175-206; 33 (1979), 339-361.

[5] Jones, V., Index for subfactors, Invent. Math., 72 (1983), 1-25.

[6] Kosaki, H., Extension of Jones' theory on index to arbitrary factors, J. Funct. Anal., 66 (1986), 123-140.

[7] Pimsner, M. and Popa, S., Entropy and index for subfactors, Ann. Sci. École Norm. Sup. Sér. 4, 19 (1986), 57-106.

[8] - Iterating the basic construction, Preprint.

[9] Strătilă, S., Modular Theory in Operator Algebras, Editura Academiei and Abacus Press, Tunbridge Wells, 1981.

[10] Umegaki, H., Conditional expectation in an operator algebra, Tôhoku Math. J., 6 (1954), 177-181. 